\title{
Observing polarimetric variability from CVs to BLAZARS
}

\section{Stephen B. Potter*}

South African Astronomical Observatory, PO Box 9, Observatory, 7935, Cape Town, South Africa

E-mail: sbpesaao.ac.za

The HIgh-speed Photo-POlarimeter of the South African Astronomical Observatory was designed and built for measuring the polarization of highly variable sources. To demonstrate its capabilities we present new detailed optical photo-polarimetric observations of two objects. Firstly of the blazar PKS 2155-304 where we recently showed the discovery of polarized quasi-periodic variations. Secondly of the recently-discovered white dwarf pulsar AR Scorpii. Our extensive dataset reveals that the polarized emission is remarkably stable and repeatable with spin, beat and orbital modulations.

Accretion Processes in Cosmic Sources - APCS2016

5-10 September 2016,

Saint Petersburg, Russia

\footnotetext{
* Speaker.
} 


\section{The polarimeter}

The HIgh-speed Photo-POlarimeter (HIPPO) of the South African Astronomical Observatory (SAAO) was designed and built for measuring the polarization of highly variable sources. It is capable of simultaneous all-Stokes, multi-filtered observations of unresolved astronomical sources. Fig. 1 (from Potter et al. 2010) shows a schematic diagram of the optical layout of the polarimeter. The wave-plates are contra-rotated at $10 \mathrm{~Hz}$ and therefore modulate the ordinary and extraordinary beams. The modulation is sufficiently rapid that errors which arise as a result of variable atmospheric conditions or telescope guiding modulations are much reduced. As both beams are modulated, each provides an independent measurement of the polarization. Therefore, simultaneous two filter observations are possible. For more details of the design see Potter et al. (2010).

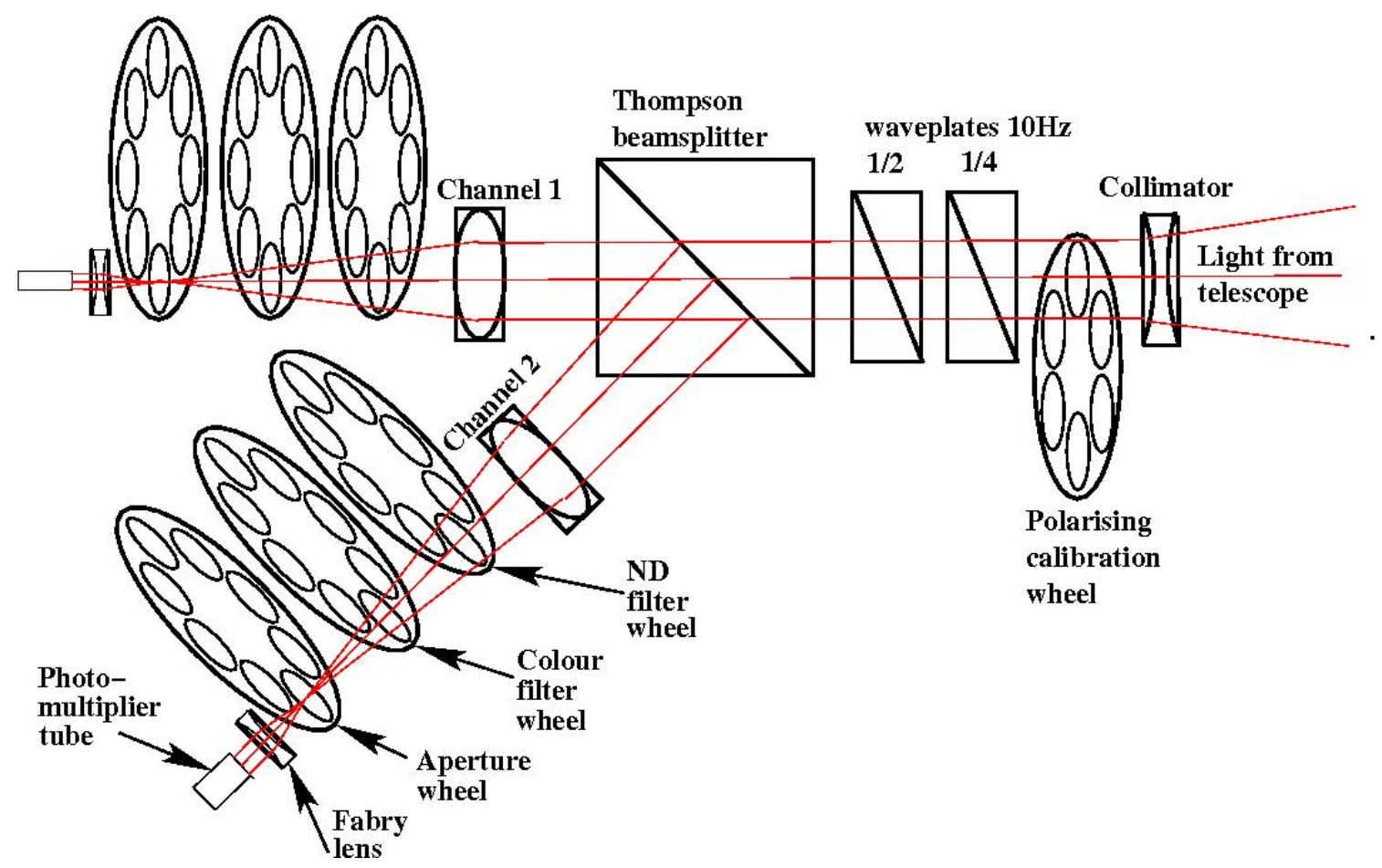

Figure 1: Optical layout of the polarimeter. Channel 1 is a copy of Channel 2.

\section{The blazar PKS 2155-304}

PKS 2155-304 is a blazar, a subclass of radio-loud active galactic nucleus for which the relativistic jet is oriented close to the line of sight of the observer (Urry \& Padovani 2009). It is powered by accretion of matter on to a supermassive black hole. The observed emission is characterized by intense and rapid brightness fluctuations across the electromagnetic spectrum which is dominated by the jet. The presence of polarization is seen at optical to radio wavelengths.

In Pekeur et al. (2016) we describe observations made with the HIPPO. In particular evidence for the presence of quasi-periodic oscillations (QPOs) in the optical polarization, during a period of enhanced gamma-ray brightness as detected by the The High Energy Stereoscopic System (H.E.S.S.). Inspection of Fig.2 (from Pekeur et al 2016) on MJD $=55037$ over the $290 \mathrm{~min}$ 
of continuous observation suggests that the amplitude of the polarization degree is modulated on a time-scale of $\sim 30 \mathrm{~min}$. The periodogram (not shown) of the polarized flux revealed the existence of a prominent peak at $\mathrm{T} \sim 13 \mathrm{~min}$, detected at $>99.7$ per cent significance, and $\mathrm{T} \sim 30 \mathrm{~min}$, which was nominally significant at $>99$ per cent. This is the first evidence of QPOs in the polarization of an active galactic nucleus, potentially opening up a new avenue of studying this phenomenon.
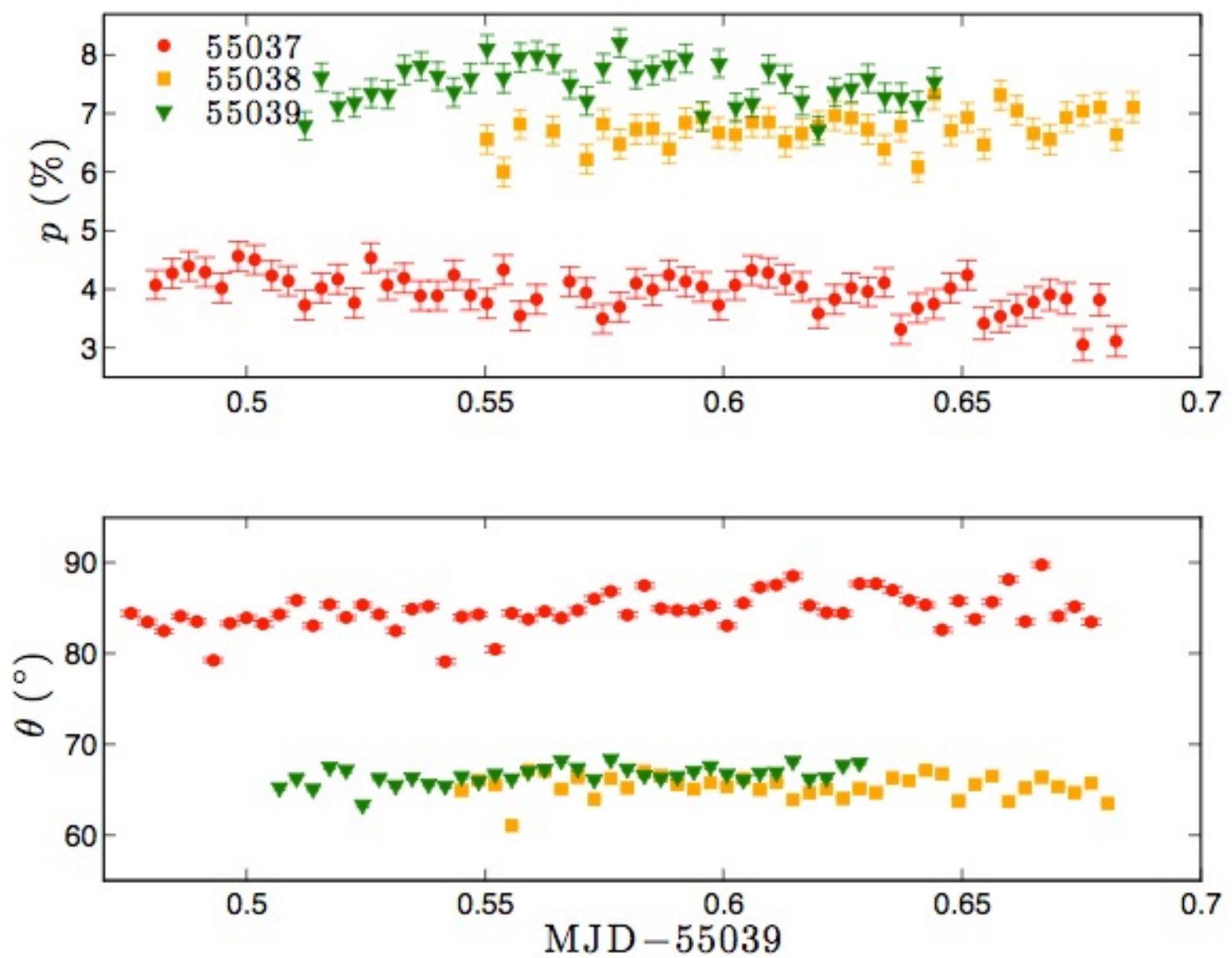

Figure 2: The intra-day variability of the I-band polarization (binned to $5 \mathrm{~min}$ ) of PKS 2155-304 on MJD $=55037$ (circles), 55038 (square markers) and 55039 (triangles). The top panel displays the polarization degree p (per cent), while the position angle of the electric vector $\theta\left(^{\circ}\right)$ is shown in the bottom panel. The polarization degree appears to be modulated by a periodic component on MJD $=55037$. From Pekeur et al. (2016)

\section{AR Sco}

AR Scorpii (hereafter AR Sco) is a 3.56h binary system consisting of a rapidly spinning $\left(P_{s}\right.$ $=117 \mathrm{~s}$ ) white dwarf and an M-type main sequence companion star. It was discovered to pulse across the electromagnetic system, from UV to radio, dominantly at the $\sim 118 \mathrm{~s}$ beat period (Marsh et al. 2016). The optical pulsed emission was discovered to be highly linearly polarized, up to $40 \%$, (Buckley et al. 2017). This led to the interpretation that AR Sco is the first white dwarf pulsar. 


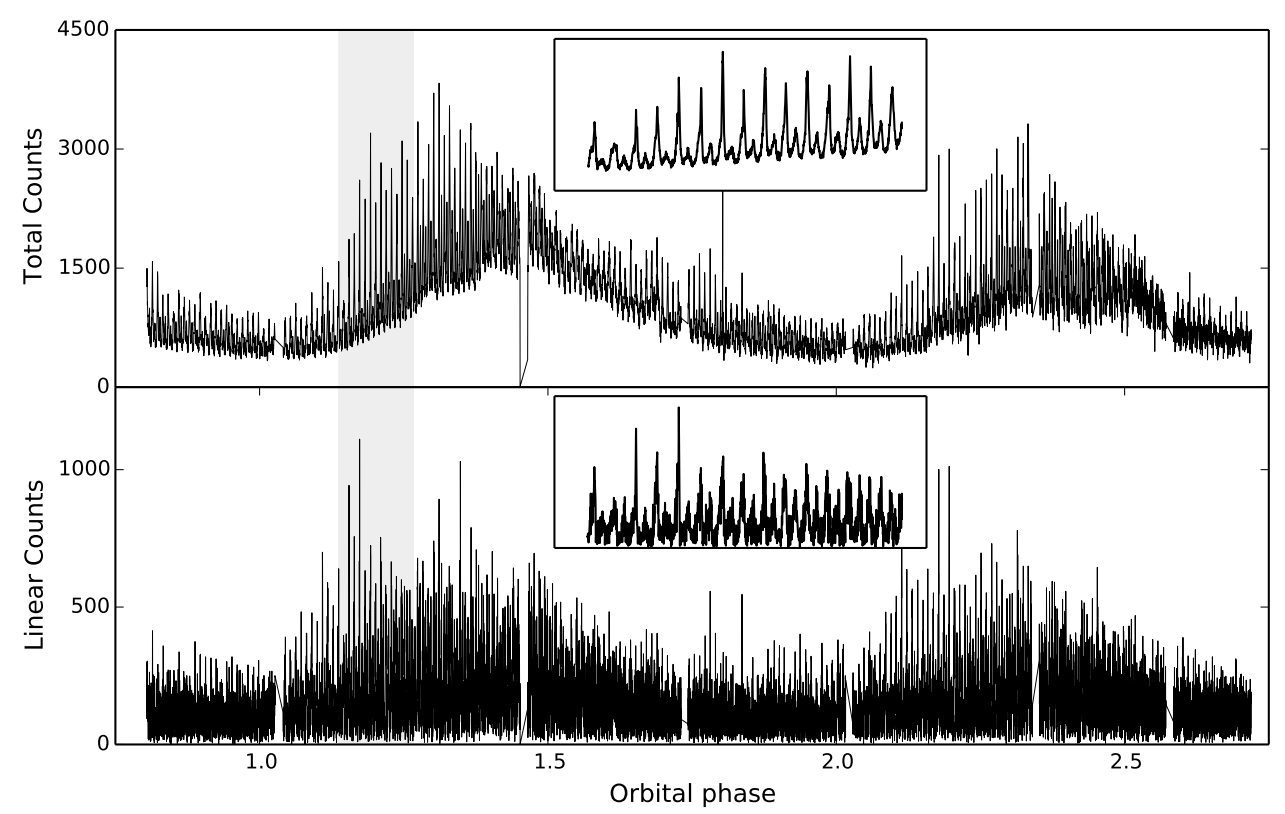

Figure 3: Top and bottom are the simultaneous photometry and polarimetry respectively. Inset show expanded views of the regions indicated in grey.

In Fig. 3. we present new high-speed photo-polarimetric observations made with the HIPPO on 27 May 2016. These are the first optical polarimetric observations covering at least one full orbital cycle. The top and bottom panels show the photometry and simultaneous linearly polarized counts respectively.

Clearly seen is the high amplitude orbital modulation covering $\sim 2.25$ cycles as well as the strong beat and spin pulses at $\sim 2$ minutes in both panels. An expanded view of part of the observations can be seen in the insets revealing the spin/beat pulses in more detail. The photometry is consistent with the observations reported by Marsh et al. (2016) and the polarimetry is consistent with the partial orbital coverage reported in Buckley et al. (2017).

In Fig.4. we show the combined amplitude spectrum of all our linear polarimetry during the 2016 and 2017 observing seasons. We are able to identify all of the peaks in the amplitude spectra. Clearly seen are the multiple harmonics of the spin $(\omega)$ and beat $(\omega-\Omega)$ frequencies as well as other sideband combinations of the spin and orbital frequencies. The spectrum is heavily aliased due to daily, weekly, monthly and yearly gaps in the dataset. The amplitude spectra is very similar to the photometric amplitude spectra (not shown).

Our extensive dataset covers multiple full orbits on several occasions over almost 2 years. The observations reveal that the polarized emission from AR Sco is remarkably repeatable between our datasets. Specifically the amplitudes of the polarized spin and beat pulses are clearly modulated as a function of orbital phase. We propose that all the polarized emission emanates from 2 diametrically opposed relativistic emission regions near the magnetic poles of the spinning white dwarf. In addition the pulsed emission is brighter at certain beat phases,i.e. the relative angle between the 


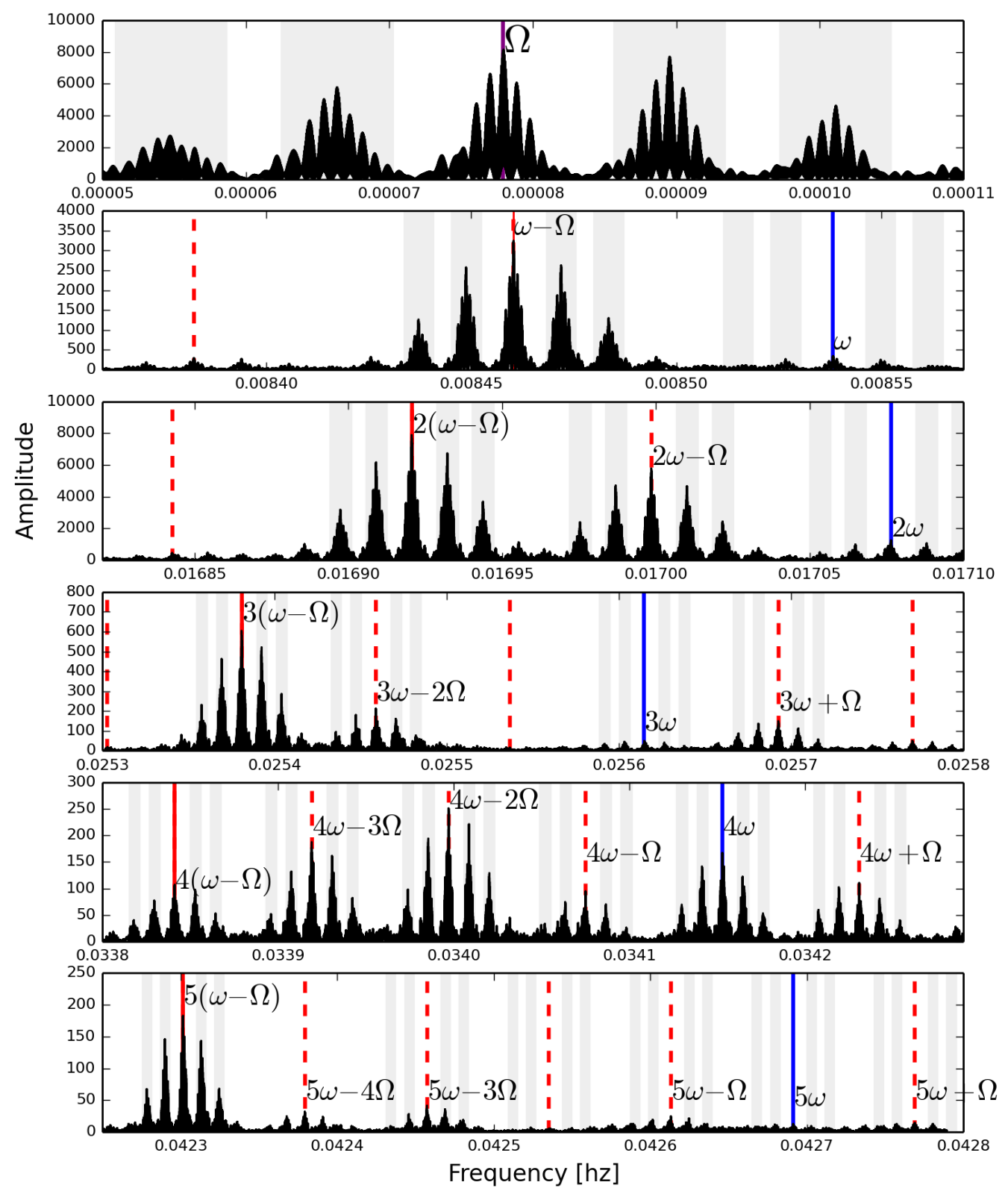

Figure 4: Amplitude spectra of the total linear flux obtained with HIPPO during the 2016 and 2017 observing seasons. Top to bottom show amplitude spectra centered on regions around the orbital frequency and the harmonics of the spin and beat frequencies. Solid blue lines indicate the spin frequency and its harmonics. Solid red lines indicate the beat frequency and its harmonics. Dashed red lines indicate other spin/orbit frequency combinations. Vertical grey bars indicate one day aliases. $\sim 15$ day aliases are also visible, particularly in the top panel. The amplitude spectra also contains $\sim 1$ year aliasing. 
white dwarf magnetic axis and the secondary star. Consequently the observer will measure larger spin pulses at certain orbital phases leading to spin, beat and orbital modulations. Details of our model will be presented elsewhere.

\section{References}

[1] Stephen B. Potter et al. Polarized QPOs from the INTEGRAL polar IGRJ14536-5522 (=Swift J1453.4-5524), 2010, MNRAS, 402, 1161

[2] Urry C. M. \& Padovani P., Unified Schemes for Radio-Loud Active Galactic Nuclei, 1995, PASP, 107, 803

[3] Pekeur et al., Evidence for quasi-periodic oscillations in the optical polarization of the blazar PKS 2155-304, 2016, MNRAS, 462, L80

[4] Marsh, T. R., et al., A radio pulsing white dwarf binary star, 2016, Nature, 537, 374

[5] Buckley, D. A. H., et al., Polarimetric evidence of a white dwarf pulsar in the binary system AR Scorpii, 2016, NatAs, 1, 29 